\title{
Effects of Puerarin Derivative P on Learning, Memory and MPO Activity in Vascular Dementia Model Mice
}

\author{
Pei Jiang ${ }^{1,2}$ and Yubin $\mathrm{Ji}^{1,2}$ \\ ${ }^{1}$ Research Center on Life Sciences and Environmental Sciences, Harbin University of Commerce,Harbin 150076,China; \\ ${ }^{2}$ Engineering Research Center of Natural Anticancer Drugs,Ministry of Education, Harbin 150076, China
}

\begin{abstract}
Pharmacological experiments confirmed that Puerarin could not only expand the arterial vessels, increase the local blood flow, but also could protect the myocardium, improve the blood supply of ischemic tissue, which was used to treat the cerebrovascular dementia disease in clinical practice.Its own structure particularity caused the lower bioavailability and poorer solubility. In order to improve the deficiency, the related pharmacological experiments of the newly synthesized puerarin derivative (P), so as to expect the better curative effect than puerarin in the process of treating vascular dementia. The vascular dementia model was established by permanently ligating the common carotid artery in mice. The effects of puerarin derivative $(\mathrm{P})$ on learning and memory in mice by water maze, Ymaze and new object discrimination methods; The myeloperoxidase activity in the ischemic cerebral cortex was evaluated in mice by biochemical method. The experimental results showed that the mice spontaneous alternation responseaccuracy in Y maze could be obviously improved in $100 \mathrm{mg} / \mathrm{kg}$ puerarin derivative group; In water maze, the swimming time to the safe platform would be significantly decreased in puerarin derivative group.Meanwhile, the MPO activity in the cerebral cortex of dementia mice was significantly decreased in $100 \mathrm{mg} / \mathrm{kg}$ puerarin derivative group by permanently ligating the unilateral common carotid artery.
\end{abstract}

Vascular dementia (VD) is a kind of intellectual disabilitydisease caused by a variety of cerebrovascular diseases (ischemic and hemorrhagic cerebrovascular disease, chronic and acute hypoxic cerebrovascular disease, etc.). It seriously damages the brain parenchyma,hinders the patients' learning ability and memory function to a certain extent, and then affects the life and health of patients[1]. At present, VD is the second common dementia disease worldwide except Alzheimer's Disease(AD) and showing a rising trendin some countries and regionsyear by year. With the continuous improvement of people's living standards, the morbidityof VD will rise perpendicularly with the increase of the age[2],[3]. Ischemic and hypoxic hypoperfusion was considered to be one of the VD pathogenesis[4].

In general, the white blood cells do not exist in the brain tissue. Theinflammatory cellsinfiltrationonly occurs at time of cerebral ischemia or hypoxia[5], [6]. Among them, the activity ofmyeloperoxidase (MPO)representing the marker of neutrophil infiltration is also increasinggradually at time of cerebral ischemia, which also proves "inflammatory response theory" in cerebral injurydue to cerebral ischemia.

Puerarinis white powdery substance extracted from the root of Radix Puerariae. Itschemical name is 7, 4'-

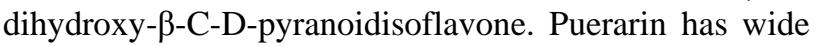
pharmacological actions, which is mainly used to treat the cardiovascular and cerebrovascular diseases [7]. Studies showed that [8], [9], puerarincould alleviatethe brain edema, remove lipid peroxidation, enhance antioxidant capacity, improve the antioxidant activity of brain tissue andalleviate focal cerebral ischemic injurydegree in cerebral ischemia reperfusion injury rats. However, the development of new drugs to treat central nervous system diseases was restricted by many factors. One of the main factors was how to effectively pass through the blood brain barrier [10]. Puerarinapplication was limited due to its lower water solubility and lipid solubility. The Puerarin structure was modified aiming to its defects in the paper and the novel puerarin derivative $\mathrm{P}$ was designed and synthesized.Its lipophilicity (logp value was increased) was increased.The effects of the new compound on learning memory functions and MPO activity were investigated preliminarily in vascular dementia mice, so as to improve the permeability of blood brain barrier, enhance its pharmacological activity, which could provide the reference for further developing and utilizingpuerarin and its derivatives in the treatment of vascular dementia.

\section{Experimental}

\subsection{Reagents and Instruments}

MS spectra were obtained with an Agilent G6300 Series (HPLC-MS) LC-MS analyzer. ${ }^{1} \mathrm{H}$ NMR spectra were obtainedwith aBruker DMX600MHz / 300MHz 
NMR spectrometer(DMSO-d6 as solvent-, tetramethylsilaneas internal standard-). AutoDockVina molecular docking software was used. Puerarin pharmaceutical raw materials $(\geqslant 98 \%)$ were purchased from Xi'an Frierson Biotechnology Co., Ltd.All reagents and solvents were of analytical grade. The silica gel used for column chromatography (reagent grade, 200 to 300 mesh)was purchased from Qingdao HaiyangChemical Co., Ltd.

\subsection{Experimental animals}

Male Kunming mice (SCXK (Army) 2013-006, weighing $25 \sim 30 \mathrm{~g}$ ) were used in this study.

\subsection{Synthesis of $P$}

Puerarin $(15 \mathrm{~g}, 36 \mathrm{mmol})$ was dissolved in anhydrous DMF (200ml) to which $\mathrm{K}_{2} \mathrm{CO}_{3}(29.8 \mathrm{~g}, 216.3 \mathrm{mmol})$ was then added at room temperature. After stirring for $90 \mathrm{~min}$, ethyl bromoacetate $(35.8 \mathrm{~g}, 216 \mathrm{mmol})$ was added and the mixture was stirred at room temperature for an additional 4h.The mixture was filtered to remove insoluble solids and then solvents were removed using a rotovapor until the mixturewas in anoily state; the oily solute was then solubilized with anhydrous ether.After again rotovappingtheether solution,the remains werespin-dried then dissolved in anhydrous methanol and isolated by silica gel column chromatography.

P(ethyl2-((3-(4-hydroxyphenyl)-4-oxo-8((2S,3R,4R,5S,6R)-3,4,5-trihydroxy-6-

(hydroxymethyl)tetrahydro-2H-pyran-2-yl)-4H-chromen7-yl)oxy)acetate) : $\quad \log \mathrm{P}-0.23$; $\quad$ ESI-MS: $\mathrm{m} / \mathrm{z} 503.1$ $[\mathrm{M}+\mathrm{H}]^{+},{ }^{1} \mathrm{H}-\mathrm{NMR}\left(300 \mathrm{MHz}, \mathrm{DMSO}-\mathrm{d}_{6}\right) \delta_{\mathrm{H}} 8.43(1 \mathrm{H}$, s,H-2), 8.07 ( $1 \mathrm{H}, \mathrm{d}, \mathrm{J}=9.0 \mathrm{~Hz}, \mathrm{H}-5), 7.18$ ( $1 \mathrm{H}, \mathrm{d}, \mathrm{J}=9.0$ Hz, H-6), 7.43 ( 1H, d, J =8.7 Hz, H-2', 6'), 6.81 ( $1 \mathrm{H}, \mathrm{d}$, $\left.\mathrm{J}=8.7 \mathrm{~Hz}, \mathrm{H}-3^{\prime}, 5^{\prime}\right), 4.88$ ( $2 \mathrm{H}, \mathrm{s}, \mathrm{H}-1^{\prime}$ ') $), 2.22$ ( $2 \mathrm{H}, \mathrm{m}$, H-3' ), 1.25 ( $3 \mathrm{H}, \mathrm{m}, \mathrm{H}-4$ '’), 4.91 ( $1 \mathrm{H}, \mathrm{d}, \mathrm{J}=9.6 \mathrm{~Hz}, \mathrm{H}-$ 1 ','), 3.95 ( $\left.1 \mathrm{H}, \mathrm{m}, \mathrm{H}-2^{\prime, ’}\right), 3.69$ ( $\left.1 \mathrm{H}, \mathrm{m}, \mathrm{H}-3{ }^{\prime \prime \prime}\right), 3.47$ ( $1 \mathrm{H}, \mathrm{m}, \mathrm{H}-4$ ', '), 3.30 ( $1 \mathrm{H}, \mathrm{m}, \mathrm{H}-5^{\prime}{ }^{\prime}$, ), 3.43 ( $1 \mathrm{H}, \mathrm{m}, \mathrm{H}-$ $\left.6 \mathrm{a}^{\prime \prime}\right), 3.65$ ( 1H, m, H-6b','), 9.55 (1H, s, -Oㅂ) $)$.

\subsection{Preparation,grouping and drug administration scheme of animal model}

The healthy male Kunming mice were intraperitoneally injected with $2 \mathrm{mg} / \mathrm{kg}$ atropine. $3.5 \%$ chloralhy drate was intraperitoneally injected $5 \mathrm{~min}$ later to anesthetize the mice. After the fixation and disinfection, the aseptic surgery was performed. The cervical median incision wasmade, the unilateral common carotid arterywas separated (not including the vagus nerve), permanently ligated and sutured with the operative thread. The mice occurred to convulsion, slow deep breathing andaccelerating heart beat. $0.3 \mathrm{ml}$ blood was discharged $1 \mathrm{~cm}$ way from the tail tip andstopped by compression. The drug was infused in the stomach $48 \mathrm{~h}$ later.The distilled water was infused in the model group and sham operation group.
The mice were randomly divided into 5 groups, 12 /group, including the sham operation group, model group, $100 \mathrm{mg} / \mathrm{kg}$ puerarinpositive group; $100 \mathrm{mg} / \mathrm{kg}$ puerarin derivative $\mathrm{P}$ group and $25 \mathrm{mg} / \mathrm{kg}$ puerarinderivative $\mathrm{P}$ group. All the mice were treated in the postoperative $48 \mathrm{~h}$. Thedistilled water was given in the sham operation group and model group.

\subsection{Measurement of MPO activity in the cerebral cortex in mice}

The mice were rapidly decapitated. The brain was removed, placed on the watch glass with ice blocks. The surface blood was sucked up. The bilateral hippocampiand cortexes wereseparated, weighed and frozen at $-80{ }^{\circ} \mathrm{C}$ for standby. The biochemical indexes were detected. $4{ }^{\circ} \mathrm{C}$ saline was added according to 1:9weight/volume ratio. $10 \%$ homogenate of brain tissue was made and centrifuged by $3000 \mathrm{r} / \mathrm{min}$ at low temperature for $15 \mathrm{~min}$. The supernatant was removed and MPO activity was detected by UV2102Cultravioletandvisiblespectrophotometeraccording to the kit.

\subsection{Statistics processing}

The experimental data were expressed using $\mathrm{x} \pm$ SD. The one-way ${ }^{[1]}$ analysis of variance was performed using SPSS18.0 statistical software. $\mathrm{P}<0.05$ indicated that the difference hadthe statistical significance.

\section{Results}

\subsection{Water maze test}

The experimentalresults showed that compared with the sham operation group, the swimming time to the safe platform was significantly prolonged in the mice of the experimental groupfrom the first day to the fifth day; Compared with the model group, the swimming time was shortened significantly in varying degrees in allPadministration groups and positive administration group. And the swimming time to the safe platform was alsodecreased significantly in the fifth day compared with that of the first day.

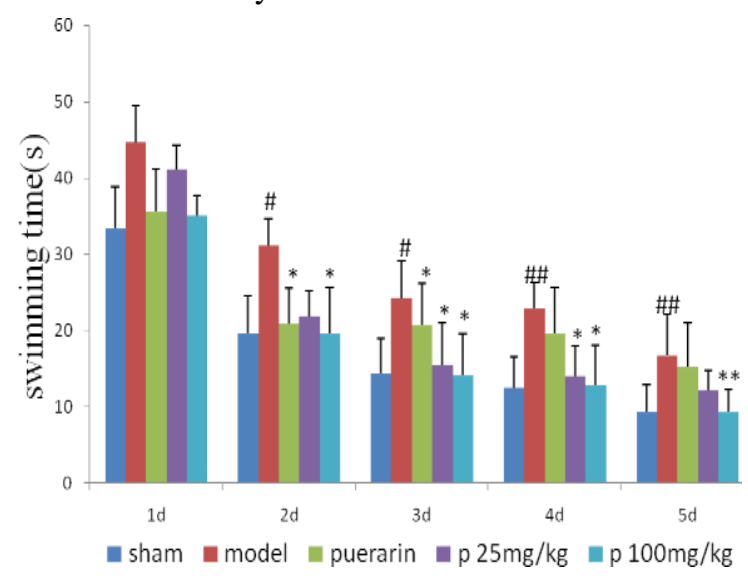


Figure 2-1. Effect of $\mathrm{P}$ on swimming time in water maze test in mice with Permanent ligation of the left common carotid artery $(\mathrm{n}=12, \mathrm{X} \pm \mathrm{SD})$

${ }^{\#} \mathrm{P}<0.05,{ }^{\# \#} \mathrm{P}<0.01$ vs. sham group; $* \mathrm{P}<0.05, * * \mathrm{P}<0.01$ vs. model group

\subsection{Y maze test}

The experimental results showed that compared with the sham operation group,the spontaneous alternating responseaccuracywas decreased significantly in the model group; Compared with the model group, themice spontaneous alternate response accuracy was increased in varying degrees in high dose $\mathrm{P}$ group, lowdose $\mathrm{P}$ group and positive drug administrationgroup.

Table 2-2. Effect of $\mathrm{P}$ on the spontaneous alternation behavior in the Y-maze test in the mice with Permanent ligation of the left common carotid artery $(n=12, \quad \bar{X} \pm S D)$

\begin{tabular}{lll}
\hline Group & Dose $(\mathrm{mg} / \mathrm{kg})$ & $\begin{array}{l}\text { Alternation } \\
\text { Behavior }(\%)\end{array}$ \\
\hline Sham & -- & $71.76 \pm 10.31$ \\
Model & -- & $41.27 \pm 8.77^{\# \#}$ \\
Puerarin & 100 & $65.21 \pm 11.44^{*}$ \\
P & 25 & $61.37 \pm 13.87$ \\
& 100 & $70.36 \pm 10.11^{* *}$
\end{tabular}

${ }^{\#} \mathrm{P}<0.01$ vs. sham group; $* \mathrm{P}<0.05,{ }^{*} * \mathrm{P}<0.01$ vs. model group.

\subsection{New object discrimination experiment}

The experimental results showed that compared with the sham operation group, the priority index and discrimination coefficient of the mice in the model group were decreased significantlyin two training periods (1h and 24h); Compared with the model group, the priority index and discrimination coefficient of the mice inhigh dose $\mathrm{P}$ group and positive drug administrationgroup were significantly enhanced in two periods ( $1 \mathrm{~h}$ and $24 \mathrm{~h}$ ).

Table 2-3. Effect of $\mathrm{P}$ on discrimination index for the new object of $1 \mathrm{~h}$ and $24 \mathrm{~h}$ in the novel object recognition in the mice with Permanent ligation of the left common carotid artery

$(\mathrm{n}=12, \quad \overline{\mathrm{X}} \pm \mathrm{SD})$

\begin{tabular}{llll}
\hline Group & Dose $(\mathrm{mg} / \mathrm{kg})$ & $\begin{array}{c}\text { Discriminati } \\
\text { on index }(1 \mathrm{~h})\end{array}$ & $\begin{array}{l}\text { Preferential } \\
\text { index }(24 \mathrm{~h})\end{array}$ \\
\hline Sham & -- & $0.47 \pm 0.08$ & $0.86 \pm 0.08$ \\
Model & -- & $0.36 \pm 0.09^{\# \#}$ & $0.46 \pm 0.06^{\# \#}$ \\
Puerari & 100 & $0.46 \pm 0.07^{*}$ & $0.76 \pm 0.09^{*}$ \\
$\mathrm{n}$ & 25 & $0.38 \pm 0.05$ & $0.66 \pm 0.06$ \\
$\mathrm{P}$ & 100 & $0.45 \pm 0.09^{*}$ & $0.81 \pm 0.11^{*}$ \\
& & & $*$ \\
\hline
\end{tabular}

${ }^{\# \#} \mathrm{P}<0.01$ vs. sham group; $* \mathrm{P}<0.05,{ }^{*} * \mathrm{P}<0.01$ vs. model group.

\subsection{Effects of puerarin derivative $P$ on MPO activity in VD mice cerebral cortex}

The results were shown in Table 2-4. Compared with the sham operation, the MPO activity in the mice cortex was significantly increased in themodel group and the inflammatory reaction wasobvious; Compared with the model group, the MPO activity was decreased in all $\mathrm{P}$ administration groups and positive drug administration group by ligating the unilateral common carotid artery.Among them, the MPO activity was significantly decreased in the high dose $\mathrm{P}$ administration group and there was significantly different.

Table 2-4. Effect of P on the activity of MPO in the mice with Permanent ligation of the left common carotid artery $\quad(n=12$, $\overline{\mathrm{X}} \pm \mathrm{SD})$

\begin{tabular}{lll}
\hline Group & Dose $(\mathrm{mg} / \mathrm{kg})$ & $\begin{array}{l}\text { MPO } \\
\text { activity }(\mathrm{U} / \mathrm{g})\end{array}$ \\
\hline Sham & -- & $0.26 \pm 0.16$ \\
Model & -- & $0.66 \pm 0.21^{\# \#}$ \\
Puerarin & 100 & $0.38 \pm 0.08^{*}$ \\
P & 25 & $0.42 \pm 0.10$ \\
& 100 & $0.36 \pm 0.15^{*}$
\end{tabular}

P $<0.01$ vs. sham group; ${ }^{*} \mathrm{P}<0.05$ vs. model group.

\section{Discussion}

In this paper ,learning and memory obstacle model was established by permanently ligating the unilateral common carotid artery. The principle of this model was to ligate the common carotid artery to causecerebral ischemia, insufficient cerebral blood supply and oxygen supply and ultimatecerebral injury. The experiments showed that the cerebral injury symptomsof the animal model were clear and definite, could cause the mice memory obstacle,could simulate the pathogenesis of VD and then could targetedly investigatethe anti-vascular dementia activity of puerarinderivative.

$\mathrm{Y}$ maze and water maze were the common behavioralapproachto study the neurodegenerative diseases. The working memory and spatial memory were investigated respectively. New object discrimination experiment was a learning and memory test method based on the principle of exploratory tendency [12], [13]. The learning and memory tests could be performed in mice under free state, and could more approximately simulate the behaviors of human learning and memory. Meanwhile, the experiment could test thelong-term or short-term memory mechanism and evaluate the memory in particular stages by flexible conversion of the new object (shape and size), which was widely used in the learning, memory, new drug evaluation and other fields. In this paper, the behavioral experiment results showed that $100 \mathrm{mg} / \mathrm{kg}$ puerarin derivative $\mathrm{P}$ could improve the learning and memory abilities of model mice in varying degrees, suggesting that it could improve the memory impairment caused by VD.

Studies [14], [15] showed that myeloperoxidase was a very important and special peroxidase secreted in neutrophil (PMN), most of which was located in PMN. The brain tissue hypoxia and ischemia would lead tothe 
inflammatory response of PMN infiltration. The myeloperoxidase (MPO) activity could indirectly reflect the PMN infiltration in cerebral ischemic areas. The cerebral cortex was the most sensitive to ischemia and was the important part of the human brain system. So the MPO activity in the cerebral cortex could indirectly reflect the degree of inflammatory damage. The experimental results showed that the MPO activity of the ischemic brain cortex was increased significantly in the model group, proved that PMN could indeed be related toa series of processes of the ischemic brain injury once again; The myeloperoxidase activity in the mice cerebral cortex could significantly decrease in high dose puerarin derivative group, so as to alleviate the inflammatory response and to improve the learning and memory abilities.

\section{Conclusions}

The structural modification of puerarin was conducted aiming to the solubility defects of puerarin, so as to obtain better lipophilic Puerarinderivatives P. $100 \mathrm{mg} / \mathrm{kg}$ puerarin derivative $\mathrm{P}$ showed better improving the learning, memory and all eviating the inflammatory response, which could provide the experimental basis to study the vascular dementia pathogenesis and therapeutic effects for natural drug structural modification.

\section{References}

1. Roman GC. Vascular dementia revisited: diagnosis, pathogenesis, treatment, and prevention. Med Clin North Am, 86(3): 477- 499(2002).

2. TorreillesF, et al[J]. Brain Res, 30: 153-163(1999)

3. LongaZ, WeinsteinPR, CarsonSeta.l Revesible middle cerebra artery without craniotomy in rats. Stroke, 20: 84- 91(1989)

4. Dohmen C,Kumura E,Rosner G,et al. Extracellular correlates of glutamate toxicity in short-term cerebral ischemia and reperfusion:a direct in vivo comparison between white and gray matter.Brain Res, 1037(1-2): 43-51(2005).

5. Poornima Venkat,Michael Chopp,Jieli Chen.Experimental Neurology.272:97-108(2005)

6. $\mathrm{Xu}$ Chen,Rong Li,Tao Liang,Kefeng Zhang,Ya Gao,Lingyuan Xu.Phytomedicine.20:849-852(2013)

7. Mohammad Abul Kaisar,Shika Prasad,Luca Cucullo.Brain Research.19(1627):90-100(2015)

8. Fatma Emel Kocak,Aysegul Kucuk,Filiz Ozyigit,Murat Tosun,Cengiz Kocak, et al.Journal of Sugical Research.199:393-401(2015)

9. Uzakov S,Frey JU, KorzV. Reinforcement of rat hippocampal LTP by hole board training. Learn -Mem.12:165-171(2005).

10. Kano T,Shimizu-Sasamata M,Huang PL,et a1.Effects of nitric oxide synthase gene knockout on neuro transmitter release in vivo.Neuro seience.86(3):695-699(2011).

11. Rosenberg GA. Ischemic brain edema. Prog Cardiovasc Dis, 42(3): 209-216(1999).

12. Luo J, Wang YH, YasudaRP, etal. Thema jority of
N2methyl2D-2aspartaterece ptorcom plexesina dultrat cerebral cortex contain at least three different subunits (NR1 /NR2A /NR2B) [J] MolPharma-col,51 (1) :79-86(1997).

13. Nathan C. Nitric oxide as a secretory product of mammalian cells.Faseb J. 6(12): 3051(1992)

14. Roman Gustavo C.Cholinergic dysfunction in vascular dementia. Curt Psychiat, Rep, 7:1826(2005).

15. HuT,Fu Q,et al.Increased acetylcholinesterase and caspase-3 expression in the brain and peripheral immune system of focal cerebral hehemicmt8. Neuroimmunol, 211 (1-2):84-91(2009). 